\title{
WEIGHTED HELLINGER DISTANCE AND IN-BETWEENNESS PROPERTY
}

\author{
Trung HoA Dinh, CONG Trinh LE*, Bich-Khue Vo \\ AND TRUnG-DUNG VuONG
}

\begin{abstract}
In this paper we introduce the weighted Hellinger distance for matrices which is an interpolating between the Euclidean distance and the Hellinger distance. We show the equivalence of the weighted Hellinger distance and the Alpha Procrustes distance. As a consequence, we prove that the matrix power mean $\mu_{p}(t, A, B)=\left(t A^{p}+(1-t) B^{p}\right)^{1 / p}$ satisfies in-betweenness property in the weighted Hellinger and Alpha Procrustes distances.
\end{abstract}

Mathematics subject classification (2010): 47A63, 47A56.

Keywords and phrases: Weighted Hellinger distance, Alpha Procrustes distance, in-betweenness property, monotonicity, in-sphere property.

\section{REFERENCES}

[1] K. M. R. AUdENAERT, In-betweenness, a geometrical monotonicity property for operator means, Linear Algebra Appl. 438 (4): 1769-1778, 2013., 16th ILAS Conference Proceedings, Pisa 2010.

[2] R. Bhatia, T. JAin, Y. Lim, On the Bures-Wasserstein distance between positive definite matrices, Expositiones Mathematicae 37 (2): 165-191, 2019.

[3] R. Bhatia, T. Jain, Y. Lim, Inequalities for the Wasserstein mean of positive definite matrices, Linear ALgerba Appl. 576 (1): 108-123, 2019.

[4] K. V. Bhagwat, R. Subramanian, Inequalities between means of positive operators, Math. Proc. Camb. Phil. Soc. 83 (5): 393-401, 1978.

[5] R. Bhatia, Y. Lim, T. YAmAZAKi, Some norm inequalities for matrix means, Linear Algebra Appl. 501: 112-122, 2016.

[6] T. H. DinH, On characterization of operator monotone functions, Linear Algebra Appl. 487: 260$267,2015$.

[7] T. H. Dinh, R. Dumitru, J. A. Franco, On the monotonicity of weighted power means for matrices, Linear Algebra Appl. 527:128-140, 2017.

[8] T. H. Dinh, R. Dumitru, J. A. Franco, Non-Linear Interpolation of the Harmonic-GeometricArithmetic Matrix Means, Lobachevskii Journal of Mathematics 40: 101-105, 2019.

[9] T. H. Dinh, R. Dumitru, J. A. Franco, Some geometric properties of matrix means with respect to different metrics, Positivity (2020), https://doi .org/10.1007/s11117-020-00738-w.

[10] T. H. DinH, B. K. T. Vo, T. Y. TAM, In-sphere property and reverse inequalities for matrix means, Elect. J. Linear Algebra 35 (1): 35-41, 2019.

[11] Q. M. HA, A Unified Formulation for the Bures-Wasserstein and Log-Euclidean/Log-Hilbert-Schmidt Distances between Positive Definite Operators, Nielsen F., Barbaresco F. (eds) Geometric Science of Information. GSI 2019. Lecture Notes in Computer Science, vol 11712. Springer, Cham, 2019.

[12] F. Kubo, T. Ando, Means of positive linear operators, Math. Ann. 246 (3): 205-224, 1980.

[13] D. SPehner, F. Illuminati, M. OrsZaG, W. RogA, Geometric measures of quantum correlations with Bures and Hellinger distances, ArXiv e-prints, November 2016.

[14] R. Bhatia, T. Jain, Y. Lim, Strong convexity of sandwiched entropies and related optimization problems, Reviews in Mathematical Physics 30 (09): 1850014, 2018. 